The Scaling and Squaring Method for the Matrix Exponential Revisited

Higham, Nicholas J.

2009

MIMS EPrint: 2009.86

Manchester Institute for Mathematical Sciences

School of Mathematics

The University of Manchester

\footnotetext{
Reports available from: http://eprints.maths.manchester.ac.uk/

And by contacting: The MIMS Secretary

School of Mathematics

The University of Manchester

Manchester, M13 9PL, UK
} 


\section{SIGEST}

The calculation of the matrix exponential $e^{A}$ may be one of the best known matrix problems in numerical computation. It achieved folk status in our community from the paper by Moler and Van Loan, "Nineteen Dubious Ways to Compute the Exponential of a Matrix," published in this journal in 1978 (and revisited in this journal in 2003). The matrix exponential is utilized in a wide variety of numerical methods for solving differential equations and many other areas.

It is somewhat amazing given the long history and extensive study of the matrix exponential problem that one can improve upon the best existing methods in terms of both accuracy and efficiency, but that is what the SIGEST selection in this issue does. "The Scaling and Squaring Method for the Matrix Exponential Revisited" by N. Higham, originally published in the SIAM Journal on Matrix Analysis and Applications in 2005, applies a new backward error analysis to the commonly used scaling and squaring method, as well as a new rounding error analysis of the Padé approximant of the scaled matrix. The analysis shows, and the accompanying experimental results verify, that a Padé approximant of a higher order than currently used actually results in a more accurate and efficient algorithm, due to the need to perform fewer matrix multiplications and fewer squarings.

SIGEST papers are expected to combine important research, broad applicability, and excellent exposition. In addition to the first two qualities that are evident from the comments above, it is no surprise that this paper exhibits the latter, since the author literally wrote the book-that is, the popular SIAM book, Handbook of Writing for the Mathematical Sciences. The paper is a true pleasure to read, and the author has added an extensive preamble for the SIGEST version that makes the topic even more accessible to new audiences as well as commenting upon subsequent work.

This paper should become an excellent choice in teaching numerical analysis as well as essential reading for researchers in numerical linear algebra. Its combination of practical algorithmic issues such as scaling, numerical analysis techniques such as backward error analysis, and a carefully conducted and presented computational study make it a true exemplar for our field. One question remains, however, after all these years: should we still assert that all the methods for calculating the matrix exponential are dubious?

The Editors 


\title{
The Scaling and Squaring Method for the Matrix Exponential Revisited*
}

Nicholas J. Higham ${ }^{\dagger}$

\begin{abstract}
The scaling and squaring method is the most widely used method for computing the matrix exponential, not least because it is the method implemented in the MATLAB function expm. The method scales the matrix by a power of 2 to reduce the norm to order 1, computes a Padé approximant to the matrix exponential, and then repeatedly squares to undo the effect of the scaling. We give a new backward error analysis of the method (in exact arithmetic) that employs sharp bounds for the truncation errors and leads to an implementation of essentially optimal efficiency. We also give a new rounding error analysis that shows the computed Padé approximant of the scaled matrix to be highly accurate. For IEEE double precision arithmetic the best choice of degree of Padé approximant turns out to be 13 , rather than the 6 or 8 used by previous authors. Our implementation of the scaling and squaring method always requires at least two fewer matrix multiplications than the expm function in MATLAB 7.0 when the matrix norm exceeds 1, which can amount to a $37 \%$ saving in the number of multiplications, and it is typically more accurate, owing to the fewer required squarings. We also investigate a different scaling and squaring algorithm proposed by Najfeld and Havel that employs a Padé approximation to the function $x \operatorname{coth}(x)$. This method is found to be essentially a variation of the standard one with weaker supporting error analysis.
\end{abstract}

Key words. matrix function, matrix exponential, Padé approximation, matrix polynomial evaluation, scaling and squaring method, MATLAB, expm, backward error analysis, performance profile

AMS subject classification. $65 \mathrm{~F} 30$

DOI. $10.1137 / 090768539$

Preamble to SIGEST Article. I remember, as a graduate student in the early 1980s, queuing up in the university library to photocopy Moler and Van Loan's "Nineteen Dubious Ways to Compute the Exponential of a Matrix" [28]. I was attracted by the intriguing title, having already had my interest in the exponential piqued by Van Loan's earlier notes [35]. The paper more than lived up to its title, and it remains for me one of the classics in numerical analysis. When I started to write Functions of Matrices [21] in 2003 the chapter on the exponential was one of the first I attempted. As I drafted a section on the scaling and squaring method, for which the basic algorithm had remained unchanged through to the 2003 update of Moler and Van Loan's paper [29], I had some difficulty in justifying the choice of parameters used in existing

${ }^{*}$ Published electronically November 6, 2009. This paper originally appeared in SIAM Journal on Matrix Analysis and Applications, Volume 26, Number 4, 2005, pages 1179-1193. This work was supported by Engineering and Physical Sciences Research Council grant GR/T08739 and by a Royal Society-Wolfson Research Merit Award.

http://www.siam.org/journals/sirev/51-4/76853.html

${ }^{\dagger}$ School of Mathematics, The University of Manchester, Manchester, M13 9PL, UK (higham@ma. man.ac.uk, http://www.ma.man.ac.uk/ higham/). 
implementations. It was through studying and reworking the analysis in [28] that I developed the ideas that led to this SIMAX article.

But what is the matrix exponential and why is it so important? The exponential of a matrix was first introduced by Laguerre [26] in 1867, who gave the now standard power series definition: for $A \in \mathbb{C}^{n \times n}$,

$$
e^{A}=I+A+\frac{A^{2}}{2 !}+\frac{A^{3}}{3 !}+\cdots .
$$

One of the first references to emphasize the important role of the matrix exponential in solving differential equations was the 1938 book Elementary Matrices and Some Applications to Dynamics and Differential Equations [13] by Frazer, Duncan, and Collar, who were in the Aerodynamics Department at the National Physical Laboratory in England. The use of $e^{A}$ in the practical solution of all kinds of differential equations is of course now ubiquitous, but other applications have come along that have no immediate connection with differential equations. I will mention just one, which is very recent.

Consider a network representing interactions between pairs of entities in a system. In recent years much work has focused on identifying computable measures that quantify characteristics of the network. Many measures are available in the literature, and they are typically expressed in terms of the network's associated undirected graph $G$ with $n$ nodes. The adjacency matrix $A \in \mathbb{R}^{n \times n}$ of the graph has $(i, j)$ element equal to 1 if nodes $i$ and $j$ are connected and 0 otherwise. Assume $a_{i i} \equiv 0$, so that there are no loops in the graph. A walk of length $m$ between two nodes $i$ and $j$ is an ordered list of nodes $i, k_{1}, k_{2}, \ldots, k_{m-1}, j$ such that successive nodes in the list are connected; the nodes need not be distinct and any of them may be $i$ or $j$. When $i=j$ the walk starts and ends at the same node and is called closed. The walk is a path if all the nodes in the walk are distinct. Assume that the graph is connected, so that a path exists between any two distinct nodes. It is a standard fact in graph theory that the $(i, j)$ element of $A^{m}$ is the number of different walks, if $i \neq j$, or closed walks, if $i=j$, of length $m$ between nodes $i$ and $j$. A variety of measures have been built by combining different walk lengths into a single number. Estrada and Rodríguez-Velázquez [11] define the subgraph centrality of node $i$ - a measure of its "well-connectedness" - by

$$
S C_{i}=\left(I+A+\frac{A^{2}}{2 !}+\frac{A^{3}}{3 !}+\cdots\right)_{i i}=\left(e^{A}\right)_{i i}
$$

By combining walks of all possible lengths connecting node $i$ to itself, and applying a weighting that decreases rapidly with the walk length, the subgraph centrality aims to capture the participation of the node in question in all subgraphs in the network. The subgraph centrality has become known as the Estrada index. Based on similar reasoning, Estrada and Hatano [8] define the communicability between nodes $i$ and $j$ - a measure of how easy it is for "information" to pass from node $i$ to node $j$ - by

$$
C_{i j}=\left(I+A+\frac{A^{2}}{2 !}+\frac{A^{3}}{3 !}+\cdots\right)_{i j}=\left(e^{A}\right)_{i j}
$$

Finally, the betweenness of node $r$ is defined by Estrada, D. J. Higham, and Hatano [10] by

$$
\frac{1}{(n-1)^{2}-(n-1)} \sum_{\substack{i, j \\ i \neq j, i \neq r, j \neq r}} \frac{\left(e^{A}-e^{A-E_{r}}\right)_{i j}}{\left(e^{A}\right)_{i j}}
$$

Copyright (C) by SIAM. Unauthorized reproduction of this article is prohibited. 
Table 0.I Some formulae for $e^{A}$.

\begin{tabular}{|c|c|c|}
\hline Power series & Limit & Scaling and squaring \\
$I+A+\frac{A^{2}}{2 !}+\frac{A^{3}}{3 !}+\cdots$ & $\lim _{s \rightarrow \infty}(I+A / s)^{s}$ & $\left(e^{A / 2^{s}}\right)^{2^{s}}$ \\
\hline Cauchy integral & Jordan form & Interpolation \\
$\frac{1}{2 \pi i} \int_{\Gamma} e^{z}(z I-A)^{-1} d z$ & $Z \operatorname{diag}\left(e^{J_{k}}\right) Z^{-1}$ & $\sum_{i=1}^{n} f\left[\lambda_{1}, \ldots, \lambda_{i}\right] \prod_{j=1}^{i-1}\left(A-\lambda_{j} I\right)$ \\
\hline Differential system & Schur form & Padé approximation \\
$Y^{\prime}(t)=A Y(t), Y(0)=I$ & $Q e^{T} Q^{*}$ & $p_{k m}(A) q_{k m}(A)^{-1}$ \\
\hline
\end{tabular}

where $E_{r}$ is zero except in row and column $r$, where it agrees with $A$. The betweenness measures the relative change in communicability when node $r$ is removed from the network. Experiments in the papers cited above show that these three measures can provide useful information about practically occurring networks that is not revealed by most other measures. In this description $A$ is symmetric, but these concepts can be extended to directed graphs, for which the adjacency matrix is unsymmetric. Of course, the matrix exponential owes its appearance to the choice of weights in the sums over walk lengths. Other weights could be chosen, resulting in different matrix functions in the definitions; see Estrada and D. J. Higham [9].

Table 0.1, taken from [21], summarizes a variety of formulae for $e^{A}$, all of which have been tried in the literature as the basis of a numerical method. The scaling and squaring method, which combines scaling and squaring with Padé approximation, has proved to be the most popular and generally applicable method.

The idea employed in this paper of adaptively choosing the degree of the Padé approximant and the amount of scaling has been taken up in subsequent work on other matrix functions, including the cosine and sine [16], [21, sect. 12.4] and the logarithm [21, sect. 11.5]. Related to the exponential are the $\psi$ functions, defined explicitly as $\psi_{k}(z)=\sum_{j=0}^{\infty} z^{j} /(j+k) !, k=0,1,2, \ldots$, or via the recurrence

$$
\psi_{k+1}(z)=\frac{\psi_{k}(z)-1 / k !}{z}, \quad \psi_{0}(z)=e^{z} .
$$

They feature in exponential integrators - a broad class of numerical methods for solving an ordinary differential equation initial value problem in which the linear part of the equation is treated exactly and the remaining nonlinear part is integrated numerically. The algorithm here has been adapted for computation of the $\psi$ functions by Koikari [25] and Skaflestad and Wright [33].

Al-Mohy and Higham have extended this work in two recent papers. The first is concerned with the Fréchet derivative $L$ at $A$ in the direction $E$, which for a general function $f$ is defined by the condition that $f(A+E)-f(A)-L(A, E)=o(\|E\|)$ for all $E$. For the exponential, the Fréchet derivative has the explicit representation

$$
L(A, E)=\int_{0}^{1} e^{A(1-s)} E e^{A s} d s .
$$

Al-Mohy and Higham [1] show how the algorithm in this paper can be "differentiated" in order to obtain an algorithm that simultaneously computes $e^{A}$ and $L(A, E)$ at a cost about three times that of computing $e^{A}$ alone. 
A weakness of the scaling and squaring method that was first pointed out by Kenney and Laub [24] is that a choice of scaling based on $\|A\|$ sometimes produces a much stronger scaling than is necessary in order to achieve the desired accuracy - with potentially detrimental consequences for numerical stability. Al-Mohy and Higham [2] show that this problem can largely be overcome by exploiting some of the information in the sequence $\left\{\left\|A^{k}\right\|\right\}$, using powers of $A$ that must be computed anyway within the algorithm along with norm estimates for a few additional choices of $k$. In some sense this is a way to deal with the effects of nonnormality of $A$. It is also shown in [2] how to exploit triangularity of $A$ to achieve better accuracy in floating point arithmetic.

Software implementing the algorithm described here is available in the function expm in MATLAB (Version 7.2, R2006a, onwards), the function MatrixExp in Mathematica (Version 5.1 onwards), and routine F01ECF in the NAG Library (from Mark 22).

Finally, it is worth emphasizing the relevance of this work for large, sparse matrices. Here, $e^{A}$ will usually be dense and it is typically $e^{A} b$ that is required in practice, for some vector $b$. Of the available methods the most developed are Krylov methods, and these require the evaluation of $e^{H}$ for a much smaller Hessenberg matrix $H$ related to $A$, for which the scaling and squaring method is well suited. For details and further references see [21, Chap. 13].

The only substantive change to this SIGEST version of the SIMAX paper was to expand slightly the description of Padé approximants and move it from section 2 to section 1.

I. Introduction. The matrix exponential is a much-studied matrix function, owing to its key role in the solution of differential equations. Computation of $e^{A}$ is required in applications such as nuclear magnetic resonance spectroscopy [17], [30], control theory [12], and Markov chain analysis [32]. Motivated by the applications, mathematicians and engineers have produced a large amount of literature on methods for computing $e^{A}$.

A wide variety of methods for computing $e^{A}$ were analyzed in the classic paper of Moler and Van Loan [28], which was reprinted with an update in [29]. The conclusion of the paper was that there are three or four candidates for best method. One of these, the scaling and squaring method, has become by far the most widely used, not least because it is the method implemented in MATLAB.

In this work we take a fresh look at the scaling and squaring method, giving a sharp analysis of truncation errors and a careful treatment of computational cost. We derive a new implementation that has essentially optimal efficiency and show that it requires at least one less matrix multiplication than existing implementations, including that in MATLAB 7.0 (R14). Our analysis and implementation are presented in section 2. Section 3 contains a comparison with existing implementations and numerical experiments. The new implementation is found to be typically more accurate than the existing ones, owing to the fact that it usually requires fewer matrix squarings. This work therefore provides another example of the phenomenon, illustrated in the work of Dhillon and Parlett [5], for example, that speed and accuracy are not always conflicting goals in matrix computations.

The scaling and squaring method employs Padé approximants to $e^{x}$. For a given scalar function $f(x)$ the rational function $r_{k m}(x)=p_{k m}(x) / q_{k m}(x)$ is a $[k / m]$ Padé approximant of $f$ [3] if $p_{k m}$ and $q_{k m}$ are polynomials of degree at most $k$ and $m$, respectively, $q_{k m}(0)=1$, and

$$
f(x)-r_{k m}(x)=O\left(x^{k+m+1}\right) .
$$


If a $[k / m]$ Padé approximant exists then it is unique. The $[k / m]$ Padé approximants to the exponential function are known explicitly for all $k$ and $m$ :

$$
p_{k m}(x)=\sum_{j=0}^{k} \frac{(k+m-j) ! k !}{(k+m) !(k-j) !} \frac{x^{j}}{j !}, \quad q_{k m}(x)=\sum_{j=0}^{m} \frac{(k+m-j) ! m !}{(k+m) !(m-j) !} \frac{(-x)^{j}}{j !} .
$$

That $r_{k m}$ satisfies the definition of Padé approximant is demonstrated by the error expression [14, Thm. 5.5.1]

$$
e^{x}-r_{k m}(x)=(-1)^{m} \frac{k ! m !}{(k+m) !(k+m+1) !} x^{k+m+1}+O\left(x^{k+m+2}\right) .
$$

Najfeld and Havel [30] propose a variation of the scaling and squaring method using Padé approximants to the function $x \operatorname{coth}(x)$, and they argue that this approach is more efficient than using direct Padé approximation of $e^{x}$. In section 4 we show that the proposed method is essentially a variation of the standard method, but with weaker supporting error analysis, both in exact arithmetic and in floating point arithmetic.

For other recent work on the scaling and squaring method, concerned particularly with arbitrary precision computations, see Sofroniou and Spaletta [34].

Throughout this paper, $\|\cdot\|$ denotes any subordinate matrix norm. We use the standard model of floating point arithmetic with unit roundoff $u$ [20, sect. 2.2]. Our rounding error bounds are expressed in terms of the constants

$$
\gamma_{k}=\frac{k u}{1-k u}, \quad \widetilde{\gamma}_{k}=\frac{c k u}{1-c k u},
$$

where $c$ denotes a small integer constant whose exact value is unimportant.

2. The Scaling and Squaring Method. The scaling and squaring method exploits the relation $e^{A}=\left(e^{A / \sigma}\right)^{\sigma}$, for $A \in \mathbb{C}^{n \times n}$ and $\sigma \in \mathbb{C}$, together with the fact that $e^{A}$ can be well approximated by a Padé approximant near the origin, that is, for small $\|A\|$. The idea is to choose $\sigma$ an integral power of $2, \sigma=2^{s}$ say, so that $A / \sigma$ has norm of order 1 ; approximate $e^{A / 2^{s}} \approx r_{k m}\left(A / 2^{s}\right)$, where $r_{k m}$ is a $[k / m]$ Padé approximant to the exponential; and then take $e^{A} \approx r_{k m}\left(A / 2^{s}\right)^{2^{s}}$, where the approximation is formed by $s$ repeated squarings. The scaling and squaring method method goes back at least to Lawson [27].

The mathematical elegance of the scaling and squaring method is enhanced by the explicit formulae (1.2). Note that $p_{k m}(x)=q_{m k}(-x)$, which reflects the property $1 / e^{x}=e^{-x}$ of the exponential function. Later we will exploit the fact that $p_{m m}(x)$ and $q_{m m}(x)$ approximate $e^{x / 2}$ and $e^{-x / 2}$, respectively, though they do so much less accurately than $r_{m m}=p_{m m} / q_{m m}$ approximates $e^{x}$. Diagonal approximants $(k=m)$ are preferred, since $r_{k m}$ with $k \neq m$ is less accurate than $r_{j j}$, where $j=\max (k, m)$, but $r_{j j}$ can be evaluated at a matrix argument at the same cost. Moreover, the diagonal approximants have the property that if the eigenvalues of $A$ lie in the open left half-plane then the eigenvalues of $r_{m m}(A)$ have modulus less than 1 (that is, the spectral radius $\rho\left(r_{m m}(A)\right)<1$ ), which is an important property in applications to differential equations [37, Chap. 8]. We will write the diagonal approximants as $r_{m}(x)=p_{m}(x) / q_{m}(x)$.

Our aim is to choose $s$, in the initial scaling $A \leftarrow A / 2^{s}$, so that the exponential is computed with backward error bounded by the unit roundoff and with minimal cost. 
In bounding the backward error we assume exact arithmetic and examine solely the effects of the approximation errors in the Padé approximant.

We begin by considering errors. The choice of $s$ will be based on $\|A\|$, where the norm can be any subordinate matrix norm. Our aim is therefore to bound the backward error in terms of $\left\|2^{-s} A\right\|$ and then to determine, for each degree $m$, the maximum $\left\|2^{-s} A\right\|$ for which $r_{m}$ can be guaranteed to deliver the desired backward error. Moler and Van Loan [28] give a very elegant backward error analysis, from which they obtain a criterion for choosing $m$; see also Golub and Van Loan [15, sect. 11.3]. Their analysis has two weaknesses. First, it makes an initial assumption that $\|A\| \leq 1 / 2$, whereas, as we will see, there are good reasons for allowing $\|A\|$ to be much larger. Second, it is designed to provide an explicit and easily computable error bound, and the resulting bound is far from being sharp. We now adapt the ideas of Moler and Van Loan in order to obtain a bound that makes no a priori assumption on $\|A\|$ and is as sharp as possible. The tradeoff is that the bound is hard to evaluate, but this is a minor inconvenience because the evaluation need only be done during the design of the algorithm.

Let

$$
e^{-A} r_{m}(A)=I+G=e^{H},
$$

where we assume that $\|G\|<1$, so that $H=\log (I+G)$ is guaranteed to exist. (Here, $\log$ denotes the principal $\operatorname{logarithm}$.) From $\log (I+G)=\sum_{j=1}^{\infty}(-1)^{j+1} G^{j} / j$, we have

$$
\|H\|=\|\log (I+G)\| \leq \sum_{j=1}^{\infty}\|G\|^{j} / j=-\log (1-\|G\|) .
$$

Now $G$ is clearly a function of $A$ (in the sense of matrix functions [21], [22, Chap. 6]), hence so is $H$, and therefore $H$ commutes with $A$. It follows that

$$
r_{m}(A)=e^{A} e^{H}=e^{A+H} .
$$

Now we replace $A$ by $A / 2^{s}$, where $s$ is a nonnegative integer, and raise both sides of this equation to the power $2^{s}$ to obtain

$$
r_{m}\left(A / 2^{s}\right)^{2^{s}}=e^{A+E},
$$

where $E=2^{s} H$ satisfies

$$
\|E\| \leq-2^{s} \log (1-\|G\|)
$$

and $G$ satisfies (2.1) with $A$ replaced by $2^{-s} A$. We summarize our findings in the following theorem.

TheOREm 2.1. Let the diagonal Padé approximant $r_{m}$ satisfy

$$
e^{-2^{-s} A} r_{m}\left(2^{-s} A\right)=I+G
$$

where $\|G\|<1$. Then

$$
r_{m}\left(2^{-s} A\right)^{2^{s}}=e^{A+E},
$$

where $E$ commutes with $A$ and

$$
\frac{\|E\|}{\|A\|} \leq \frac{-\log (1-\|G\|)}{\left\|2^{-s} A\right\|}
$$


Theorem 2.1 is a backward error result: it interprets the truncation errors in the Padé approximant as equivalent to a perturbation in the original matrix $A$. (The result holds, in fact, for any rational approximation $r_{m}$, as we have not yet used specific properties of a Padé approximant.) The advantage of the backward error viewpoint is that it automatically takes into account the effect of the squaring phase on the error in the Padé approximant and, compared with a forward error bound, avoids the need to consider the conditioning of the problem.

Our task now is to bound the norm of $G$ in (2.2) in terms of $\left\|2^{-s} A\right\|$. Define the function

$$
\rho(x)=e^{-x} r_{m}(x)-1 .
$$

In view of the Padé approximation property (1.3), $\rho$ has a power series expansion

$$
\rho(x)=\sum_{i=2 m+1}^{\infty} c_{i} x^{i}
$$

and this series will converge absolutely for $|x|<\min \left\{|t|: q_{m}(t)=0\right\}=: \nu_{m}$. Hence

$$
\|G\|=\left\|\rho\left(2^{-s} A\right)\right\| \leq \sum_{i=2 m+1}^{\infty}\left|c_{i}\right| \theta^{i}=: f(\theta)
$$

where $\theta:=\left\|2^{-s} A\right\|<\nu_{m}$. It is clear that if $A$ is a general matrix and only $\|A\|$ is known then (2.5) provides the smallest possible bound on $\|G\|$. The corresponding bound of Moler and Van Loan [28, Appx. 1, Lem. 4] is easily seen to be less sharp, and a refined analysis of Dieci and Papini [6, sect. 2], which bounds a different error, is also weaker when adapted to bound $\|G\|$.

Combining (2.5) with (2.3) we have

$$
\frac{\|E\|}{\|A\|} \leq \frac{-\log (1-f(\theta))}{\theta}
$$

Evaluation of $f(\theta)$ in (2.5) would be easy if the coefficients $c_{i}$ were one-signed, for then we would have $f(\theta)=|\rho(\theta)|$. Experimentally, the $c_{i}$ are one-signed for some, but not all, $m$. Using MATLAB with the Symbolic Math Toolbox we have evaluated $f(\theta)$, and hence the bound (2.6), in 250 decimal digit arithmetic, summing the first 150 terms of the series, where the $c_{i}$ in (2.4) are obtained symbolically. For $m=1: 21$ we have used a zero-finder to determine the largest value of $\theta$, denoted by $\theta_{m}$, such that the backward error bound (2.6) does not exceed $u=2^{-53} \approx 1.1 \times 10^{-16}$, the unit roundoff in IEEE double precision arithmetic. The results are shown to two significant figures in Table 2.1.

The second row of the table shows the values of $\nu_{m}$, and we see that $\theta_{m}<\nu_{m}$ in each case, confirming that the bound (2.5) is valid. The inequalities $\theta_{m}<\nu_{m}$ also confirm the important fact that $q_{m}(A)$ is nonsingular for $\|A\| \leq \theta_{m}$ (which is in any case implicitly enforced by our analysis).

Next we need to determine the cost of evaluating $r_{m}(A)$. Because of the relation $q_{m}(x)=p_{m}(-x)$ between the numerator and denominator polynomials, an efficient scheme can be based on explicitly computing the even powers of $A$, forming $p_{m}$ and $q_{m}$, and then solving the matrix equation $q_{m} r_{m}=p_{m}$ [36]. If $p_{m}(x)=\sum_{i=0}^{m} b_{i} x^{i}$, we have, for the even-degree case,

$$
\begin{aligned}
p_{2 m}(A) & =b_{2 m} A^{2 m}+\cdots+b_{2} A^{2}+b_{0} I+A\left(b_{2 m-1} A^{2 m-2}+\cdots+b_{3} A^{2}+b_{1} I\right) \\
& =: U+V
\end{aligned}
$$

Copyright (c) by SIAM. Unauthorized reproduction of this article is prohibited. 
Table 2.I Maximal values $\theta_{m}$ of $\left\|2^{-s} A\right\|$ such that the backward error bound (2.6) does not exceed $u=2^{-53}$, values of $\nu_{m}=\min \left\{|x|: q_{m}(x)=0\right\}$, and upper bound $\xi_{m}$ for $\left\|q_{m}(A)^{-1}\right\|$.

\begin{tabular}{|c|c|c|c|c|c|c|c|c|c|c|c|}
\hline$m$ & 1 & 2 & 3 & 4 & 5 & 6 & 7 & 8 & 9 & 10 & \\
\hline$\theta_{m}$ & $3.7 \mathrm{e}-8$ & $5.3 \mathrm{e}-4$ & $1.5 \mathrm{e}-2$ & $8.5 \mathrm{e}-2$ & $2.5 \mathrm{e}-1$ & $5.4 \mathrm{e}-1$ & $9.5 \mathrm{e}-1$ & $1.5 \mathrm{e} 0$ & $2.1 \mathrm{e} 0$ & $2.8 \mathrm{e} 0$ & \\
\hline$\nu_{m}$ & $2.0 \mathrm{e} 0$ & $3.5 \mathrm{e} 0$ & $4.6 \mathrm{e} 0$ & $6.0 \mathrm{e} 0$ & $7.3 \mathrm{e} 0$ & $8.7 \mathrm{e} 0$ & $9.9 \mathrm{e} 0$ & 1.1e1 & 1.3e1 & $1.4 \mathrm{e} 1$ & \\
\hline$\xi_{m}$ & $1.0 \mathrm{e} 0$ & $1.0 \mathrm{e} 0$ & $1.0 \mathrm{e} 0$ & $1.0 \mathrm{e} 0$ & $1.1 \mathrm{e} 0$ & $1.3 \mathrm{e} 0$ & $1.6 \mathrm{e} 0$ & $2.1 \mathrm{e} 0$ & $3.0 \mathrm{e} 0$ & $4.3 \mathrm{e} 0$ & \\
\hline$m$ & 11 & 12 & 13 & 14 & 15 & 16 & 17 & 18 & 19 & 20 & 21 \\
\hline$\theta_{m}$ & $3.6 \mathrm{e} 0$ & $4.5 \mathrm{e} 0$ & $5.4 \mathrm{e} 0$ & $6.3 \mathrm{e} 0$ & 7.3e0 & $8.4 \mathrm{e} 0$ & $9.4 \mathrm{e} 0$ & 1.1e1 & $1.2 \mathrm{e} 1$ & $1.3 \mathrm{e} 1$ & $1.4 \mathrm{e} 1$ \\
\hline$\nu_{m}$ & $1.5 \mathrm{e} 1$ & $1.7 \mathrm{e} 1$ & $1.8 \mathrm{e} 1$ & $1.9 \mathrm{e} 1$ & $2.1 \mathrm{e} 1$ & $2.2 \mathrm{e} 1$ & 2.3e1 & $2.5 \mathrm{e} 1$ & $2.6 \mathrm{e} 1$ & $2.7 \mathrm{e} 1$ & $2.8 \mathrm{e} 1$ \\
\hline$\xi_{m}$ & $6.6 \mathrm{e} 0$ & $1.0 \mathrm{e} 1$ & $1.7 \mathrm{e} 1$ & $3.0 \mathrm{e} 1$ & $5.3 \mathrm{e} 1$ & $9.8 \mathrm{e} 1$ & $1.9 \mathrm{e} 2$ & $3.8 \mathrm{e} 2$ & 8.3e2 & $2.0 \mathrm{e} 3$ & $6.2 \mathrm{e} 3$ \\
\hline
\end{tabular}

Table 2.2 Number of matrix multiplications, $\pi_{m}$, required to evaluate $p_{m}(A)$ and $q_{m}(A)$, and the measure of overall cost $C_{m}$ in (2.10).

\begin{tabular}{r|ccccccccccc}
\hline$m$ & 1 & 2 & 3 & 4 & 5 & 6 & 7 & 8 & 9 & 10 & \\
\hline$\pi_{m}$ & 0 & 1 & 2 & 3 & 3 & 4 & 4 & 5 & 5 & 6 & \\
$C_{m}$ & 25 & 12 & 8.1 & 6.6 & 5.0 & 4.9 & 4.1 & 4.4 & 3.9 & 4.5 & \\
\hline \multicolumn{1}{l}{} & & & & & & & & & & \\
\hline$m$ & 11 & 12 & 13 & 14 & 15 & 16 & 17 & 18 & 19 & 20 & 21 \\
\hline$\pi_{m}$ & 6 & 6 & 6 & 7 & 7 & 7 & 7 & 8 & 8 & 8 & 8 \\
$C_{m}$ & 4.2 & 3.8 & 3.6 & 4.3 & 4.1 & 3.9 & 3.8 & 4.6 & 4.5 & 4.3 & 4.2 \\
\hline
\end{tabular}

which can be evaluated with $m+1$ matrix multiplications by forming $A^{2}, A^{4}, \ldots$, $A^{2 m}$. Then

$$
q_{2 m}(A)=U-V
$$

is available at no extra cost. For odd degrees,

$$
\begin{aligned}
p_{2 m+1}(A) & =A\left(b_{2 m+1} A^{2 m}+\cdots+b_{3} A^{2}+b_{1} I\right)+b_{2 m} A^{2 m}+\cdots+b_{2} A^{2}+b_{0} I \\
& =: U+V
\end{aligned}
$$

and so $p_{2 m+1}$ and $q_{2 m+1}=-U+V$ can be evaluated at exactly the same cost as $p_{2 m}$ and $q_{2 m}$. However, for $m \geq 12$ this scheme can be improved upon. For example, we can write

$$
\begin{aligned}
p_{12}(A)= & A^{6}\left(b_{12} A^{6}+b_{10} A^{4}+b_{8} A^{2}+b_{6} I\right)+b_{4} A^{4}+b_{2} A^{2}+b_{0} I \\
& +A\left[A^{6}\left(b_{11} A^{4}+b_{9} A^{2}+b_{7} I\right)+b_{5} A^{4}+b_{3} A^{2}+b_{1} I\right] \\
= & : U+V
\end{aligned}
$$

and $q_{12}(A)=U-V$. Thus $p_{12}$ and $q_{12}$ can be evaluated in just six matrix multiplications (for $A^{2}, A^{4}, A^{6}$, and three additional multiplications). For $m=13$ an analogous formula holds with the outer multiplication by $A$ transferred to the $U$ term. Similar formulae hold for $m \geq 14$. Table 2.2 summarizes the number of matrix multiplications required to evaluate $p_{m}$ and $q_{m}$, which we denote by $\pi_{m}$, for $m=1: 21$.

The information in Tables 2.1 and 2.2 enables us to determine the optimal algorithm when $\|A\| \geq \theta_{21}$. From Table 2.2, we see that the choice is between $m=1,2,3$, $5,7,9,13,17$, and 21 . (There is no reason to use $m=6$, for example, since the cost of evaluating the more accurate $q_{7}$ is the same as the cost of evaluating $q_{6}$.) Increasing from one of these values of $m$ to the next requires an extra matrix multiplication to 
evaluate $r_{m}$, but this is offset by the larger allowed $\theta_{m}=\left\|2^{-s} A\right\|$ if $\theta_{m}$ jumps by more than a factor 2 , since decreasing $s$ by 1 saves one multiplication in the final squaring stage. Table 2.1 therefore shows that $m=13$ is the best choice. Another way to arrive at this conclusion is to observe that the cost of the algorithm in matrix multiplications is, since $s=\left\lceil\log _{2}\|A\| / \theta_{m}\right\rceil$ if $\|A\| \geq \theta_{m}$ and $s=0$ otherwise,

$$
\pi_{m}+s=\pi_{m}+\max \left(\left\lceil\log _{2}\|A\|-\log _{2} \theta_{m}\right\rceil, 0\right) .
$$

(We ignore the required matrix equation solution, which is common to all $m$.) We wish to determine which $m$ minimizes this quantity. For $\|A\| \geq \theta_{m}$ we can remove the max and ignore the $\|A\|$ term, which is essentially a constant shift, and so we minimize

$$
C_{m}=\pi_{m}-\log _{2} \theta_{m}
$$

The $C_{m}$ values are shown in the second line of Table 2.2. Again, $m=13$ is clearly the best choice. We repeated the computations with $u=2^{-24} \approx 6.0 \times 10^{-8}$, which is the unit roundoff in IEEE single precision arithmetic, and $u=2^{-105} \approx 2.5 \times 10^{-32}$, which corresponds to quadruple precision arithmetic; the optimal $m$ are now $m=7$ and $m=17$, respectively.

Now we consider the effects of rounding errors on the evaluation of $r_{m}(A)$. We immediately rule out $m=1$ and $m=2$ because $r_{1}$ and $r_{2}$ can suffer from loss of significance in floating point arithmetic. For example, $r_{1}$ requires $\|A\|$ to be of order $10^{-8}$ after scaling, and then the expression $r_{1}(A)=(I+A / 2)(I-A / 2)^{-1}$ loses about half the significant digits in $A$ in double precision arithmetic; yet if the original $A$ has norm of order at least 1 then all the significant digits of some of the elements of $A$ should contribute to the result.

The effect of rounding errors on the evaluation of the numerator and denominator of $r_{m}(A)$ is described by the following result, which can be proved using techniques from $[20]$.

THEOREM 2.2. Let $g_{m}(x)=\sum_{k=0}^{m} b_{k} x^{k}$. The computed polynomial $\widehat{g}_{m}$ obtained by evaluating $g_{m}$ at $X \in \mathbb{C}^{n \times n}$ using explicit formation of matrix powers as in the methods above satisfies

$$
\left|g_{m}-\widehat{g}_{m}\right| \leq \widetilde{\gamma}_{m n} \widetilde{g}_{m}(|X|),
$$

where $\widetilde{g}_{m}(X)=\sum_{i=0}^{m}\left|b_{k}\right| X^{k}$. Hence $\left\|g_{m}-\widehat{g}_{m}\right\|_{1} \leq \widetilde{\gamma}_{m n} \widetilde{g}_{m}\left(\|X\|_{1}\right)$.

Applying the theorem to $p_{m}(A)$, where $\|A\|_{1} \leq \theta_{m}$, and noting that $p_{m}$ has all positive coefficients, we deduce that

$$
\begin{aligned}
\left\|p_{m}(A)-\widehat{p}_{m}(A)\right\|_{1} & \leq \widetilde{\gamma}_{m n} p_{m}\left(\|A\|_{1}\right) \\
& \approx \widetilde{\gamma}_{m n} e^{\|A\|_{1} / 2} \\
& \leq \widetilde{\gamma}_{m n}\left\|e^{A / 2}\right\|_{1} e^{\|A\|_{1}} \\
& \approx \widetilde{\gamma}_{m n}\left\|p_{m}(A)\right\|_{1} e^{\|A\|_{1}} \leq \widetilde{\gamma}_{m n}\left\|p_{m}(A)\right\|_{1} e^{\theta_{m}} .
\end{aligned}
$$

Hence the relative error is bounded approximately by $\widetilde{\gamma}_{m n} e^{\theta_{m}}$, which is a very satisfactory bound, given the values of $\theta_{m}$ in Table 2.1. Replacing $A$ by $-A$ in the latter bound we obtain

$$
\left\|q_{m}(A)-\widehat{q}_{m}(A)\right\|_{1} \lesssim \widetilde{\gamma}_{m n}\left\|q_{m}(A)\right\|_{1} e^{\theta_{m}}
$$


Table 2.3 Constants $\theta_{m}$ needed in Algorithm 2.3.

\begin{tabular}{r|c}
\hline$m$ & $\theta_{m}$ \\
\hline 3 & $1.495585217958292 \mathrm{e}-2$ \\
5 & $2.539398330063230 \mathrm{e}-1$ \\
7 & $9.504178996162932 \mathrm{e}-1$ \\
9 & $2.097847961257068 \mathrm{e} 0$ \\
13 & $5.371920351148152 \mathrm{e} 0$ \\
\hline
\end{tabular}

In summary, the errors in the evaluation of $p_{m}$ and $q_{m}$ are nicely bounded. This analysis improves that of Ward [38, eq. (3.5)], who assumes $\|A\| \leq 1$ and obtains absolute error bounds.

To obtain $r_{m}$ we solve a multiple right-hand side linear system with $q_{m}(A)$ as coefficient matrix, so to be sure that this system is solved accurately we need to check that $q_{m}(A)$ is well conditioned. It is possible to obtain a priori bounds for $\left\|q_{m}(A)^{-1}\right\|$ under assumptions such as $\|A\| \leq 1 / 2$ [28, Appx. 1, Lem. 2], $\|A\| \leq 1$ [38, Thm. 1], or $q_{m}(-\|A\|)<2$ [6, Lem. 2.1], but these assumptions are not satisfied for all the $m$ and $\|A\|$ of interest to us. Therefore we take a similar approach to the way we derived the constants $\theta_{m}$. With $\|A\| \leq \theta_{m}$ and by writing

$$
\left.q_{m}(A)=e^{-A / 2}\left(I+e^{A / 2} q_{m}(A)-I\right)\right) \equiv e^{-A / 2}(I+F),
$$

we have, if $\|F\|<1$,

$$
\left\|q_{m}(A)^{-1}\right\| \leq\left\|e^{A / 2}\right\|\left\|(I+F)^{-1}\right\| \leq \frac{e^{\theta_{m} / 2}}{1-\|F\|} .
$$

We can expand $e^{x / 2} q_{m}(x)-1=\sum_{i=2}^{\infty} d_{i} x^{i}$, from which $\|F\| \leq \sum_{i=2}^{\infty}\left|d_{i}\right| \theta_{m}^{i}$ follows. Our overall bound is

$$
\left\|q_{m}(A)^{-1}\right\| \leq \frac{e^{\theta_{m} / 2}}{1-\sum_{i=2}^{\infty}\left|d_{i}\right| \theta_{m}^{i}} .
$$

By determining the $d_{i}$ symbolically and summing the first 150 terms of the sum in 250 decimal digit arithmetic, we obtained the bounds in the last row of Table 2.1, which confirm that $q_{m}$ is very well conditioned for $m$ up to about 13 when $\|A\| \leq \theta_{m}$.

Our algorithm is as follows. It first checks whether $\|A\| \leq \theta_{m}$ for $m \in\{3,5,7$, $9,13\}$ and, if so, evaluates $r_{m}$ for the smallest such $m$. Otherwise it uses the scaling and squaring method with $m=13$.

Algorithm 2.3. This algorithm evaluates the matrix exponential of $A \in \mathbb{C}^{n \times n}$ using the scaling and squaring method. It uses the constants $\theta_{m}$ given in Table 2.3.

$1 \%$ Coefficients of degree 13 Padé approximant.

$2 b(0: 13)=[64764752532480000,32382376266240000,7771770303897600$,

$3 \quad 1187353796428800,129060195264000,10559470521600$,

$4 \quad 670442572800,33522128640,1323241920$,

$5 \quad 40840800,960960,16380,182,1]$

$6 \%$ Preprocessing to reduce the norm.

$7 \quad A \leftarrow A-\mu I$, where $\mu=\operatorname{trace}(A) / n$.

$8 A \leftarrow D^{-1} A D$, where $D$ is a balancing transformation (or set $D=I$ if balancing does not reduce the 1-norm of $A$ ).

9 for $m=\left[\begin{array}{lllll}3 & 5 & 7 & 9 & 13\end{array}\right]$ 


$$
\begin{aligned}
& \text { if }\|A\|_{1} \leq \theta_{m} \\
& X=r_{m}(A) \% r_{m}(A)=[m / m] \text { Padé approximant to } A \text {. } \\
& X=e^{\mu} D X D^{-1} \% \text { Undo preprocessing. } \\
& \text { end }
\end{aligned}
$$

The cost of Algorithm 2.3 is $\pi_{m}+\left\lceil\log _{2}\left(\|A\|_{1} / \theta_{m}\right)\right\rceil$ matrix multiplications, where $m$ is the degree of Padé approximant used, and $\pi_{m}$ is tabulated in Table 2.2, plus the solution of one matrix equation.

It is readily checked that the sequences $\theta_{13}^{2 k} b_{2 k}$ and $\theta_{13}^{2 k+1} b_{2 k+1}$ are approximately monotonically decreasing with $k$, and hence the ordering given in Algorithm 2.3 for evaluating $U$ and $V$ takes the terms in approximately increasing order of norm. This ordering is certainly preferable when $A$ has nonnegative elements, and since there cannot be much cancellation in the sums it cannot be a bad ordering [20, Chap. 4].

The Padé approximant $r_{m}$ at line 11 is intended to be evaluated using (2.8) for $m \leq 9$, or as in lines $17-19$ for $m=13$.

The preprocessing in Algorithm 2.3 is precisely that suggested by Ward [38] and attempts to reduce the norm by a shift and a similarity transformation.

The use of the [13/13] Padé approximation in Algorithm 2.3 gives optimal efficiency. However, Table 2.1 reports a bound of 3.0 for $\left\|q_{9}(A)^{-1}\right\|$, which is somewhat smaller than the bound of 17 for $\left\|q_{13}(A)^{-1}\right\|$, and $C_{9}$ is only slightly larger than $C_{13}$; therefore the best compromise between numerical stability and efficiency could conceivably be obtained by limiting to maximum degree $m=9$. We will compare these two degrees experimentally in the next section.

3. Comparison with Existing Algorithms. We now compare Algorithm 2.3 with existing implementations of the scaling and squaring method that also employ Padé approximations to $e^{x}$.

The function expm in MATLAB 7.0 (R14) uses $m=6$ with $\left\|2^{-s} A\right\|_{\infty} \leq 0.5$ as the scaling criterion and does not employ preprocessing. (expm is a built-in function, but expmdemo1 $1^{1}$ is an M-file implementation of the same algorithm, and in all our tests expm and expmdemo1 produced exactly the same results.) Sidje [31] uses the same parameters in his function padm. Surprisingly, neither expm nor padm evaluates $r_{6}$ optimally: whereas (2.7) requires just 4 multiplications, expm uses 5 , because it evaluates all the powers $A^{2}, A^{3}, \ldots, A^{6}$, while padm expends 7 multiplications in using Horner's method with a variant of (2.7). We note that in padm, $p_{m}$ and $q_{m}$ are evaluated in increasing order of norms of the terms, as in Algorithm 2.3, whereas expm uses the reverse ordering.

Ward [38] uses $m=8$ with $\left\|2^{-s} A\right\|_{1} \leq 1$ and carries out the same preprocessing as Algorithm 2.3.

\footnotetext{
${ }^{1}$ As of MATLAB 7.8 (R2009a), expmdemo1 remains unchanged.
} 
In the following discussion we will assume that all the algorithms use the same norm and ignore the preprocessing.

Since Ward's value of $\theta$ is twice that used in expm and padm, and the [8/8] Padé approximant can be evaluated with just one more matrix multiplication than the $[6 / 6]$ one, Ward's algorithm would have exactly the same cost as expm and padm for $\|A\| \geq 0.5$, were the latter algorithms to evaluate $r_{6}$ efficiently.

It is clear from our analysis that the three algorithms under discussion are not of optimal efficiency. If the [6/6] or [8/8] Padé approximants are to be used, then one can take larger values of $\theta$, as shown by Table 2.1. Moreover, as we have argued, there is no reason to use the degree 6 or 8 approximants because the degree 7 and 9 approximants have the same cost, respectively.

By considering Tables 2.1 and 2.2 it is easy to see the following:

- When $\|A\|_{1}>1$, Algorithm 2.3 requires one or two fewer matrix multiplications than Ward's implementation, two or three fewer than expm, and four or five fewer than padm. For example, when $\|A\|_{1} \in(2,2.1)$, the number of matrix multiplications reduces from 8 for expm and 7 for Ward's implementation to 5 for Algorithm 2.3 (which takes $m=9$ ) - a saving of $37 \%$ and $29 \%$, respectively.

- When $\|A\|_{1} \leq 1$, Algorithm 2.3 requires no more matrix multiplications than expm, padm, and Ward's algorithm, and up to 3,5 , and 3 fewer, respectively.

Our analysis shows that all these algorithms have a backward error no larger than $u$, ignoring roundoff. However, it is well known that rounding errors can significantly affect the scaling and squaring method, because the squaring phase can suffer from severe numerical cancellation. The fundamental problem can be seen in the result [20, sect. 3.5]

$$
\left\|A^{2}-f l\left(A^{2}\right)\right\|_{1} \leq \gamma_{n}\|A\|_{1}^{2},
$$

which shows that the errors in the computed squared matrix are small compared with the square of the norm of the original matrix but not necessarily small compared with the matrix being computed. By using standard error analysis techniques it is possible to derive a forward error bound for the scaling and squaring method, as has been done by Ward [38]. However, with our current knowledge of the $e^{A}$ problem it is not easy to determine whether a large value for the bound signals potential instability of the method or an ill-conditioned problem.

Since the matrix squarings in the scaling and squaring method are potentially dangerous it seems desirable to minimize the number of them. Algorithm 2.3 uses one to three fewer squarings than the algorithms with which we have compared it, and hence it has a potential advantage in accuracy.

We now present some numerical experiments, carried out in MATLAB 7.0 (R14), that provide some insight into the accuracy of the scaling and squaring method and of Algorithm 2.3. We took $668 \times 8$ test matrices: 53 obtained from the function matrix in the Matrix Computation Toolbox [19] (which include test matrices from MATLAB itself), together with 13 further test matrices of dimension 2-10 from [4, Ex. 3], [6, Ex. 3.10], [24, Ex. 2 and p. 655], [30, p. 370], and [38, Test Cases 1-4]. We evaluated the relative error in the 1-norm of the computed matrices from expm, from Algorithm 2.3, and from a modified version of Algorithm 2.3 in which the maximal degree of the Padé approximant is a parameter, $m_{\max }$. The latter algorithm, denoted by $\operatorname{Exp}\left(m_{\max }\right)$, allows us to study the dependence of the error on $m_{\max }$. We did not use any preprocessing in this experiment, although we found that turning on 


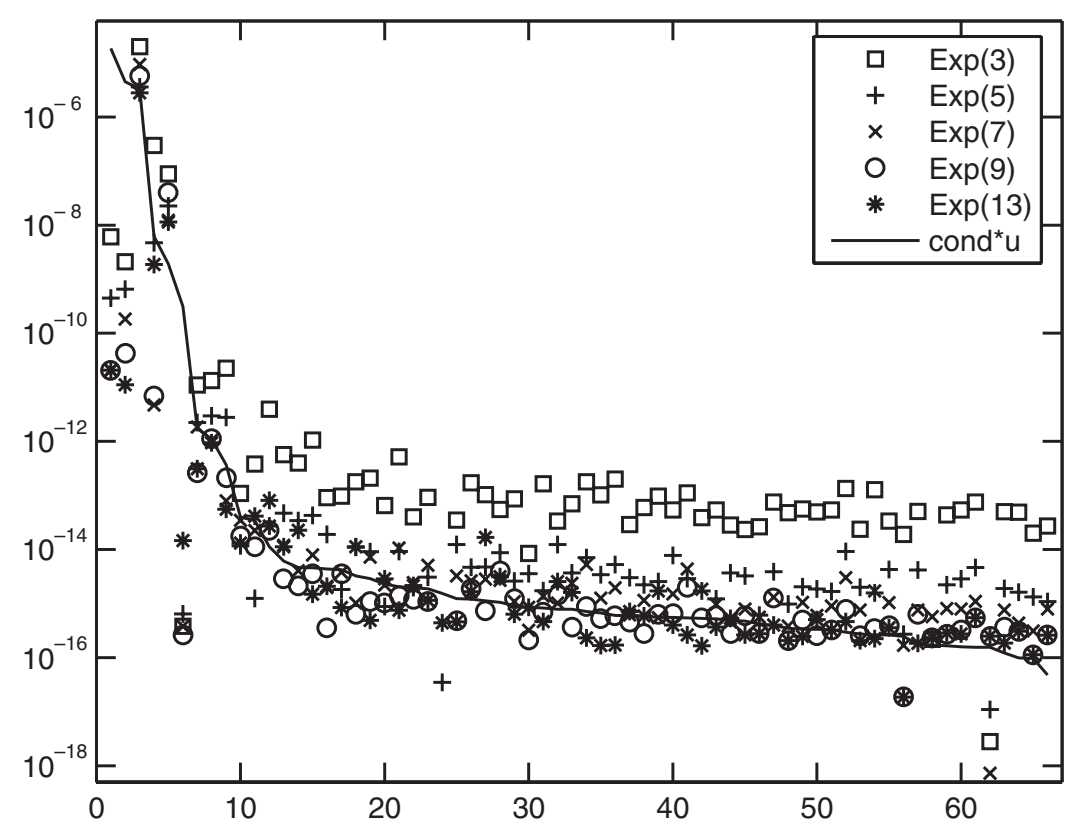

Fig. 3.I Normwise relative errors for Algorithm $2.3(\operatorname{Exp}(13))$ and variants with $m_{\max }$ restricted to $3,5,7$, and 9 .

preprocessing in Algorithm 2.3 makes essentially no difference to the results. The "exact" $e^{A}$ is obtained at 100-digit precision using the Symbolic Math Toolbox.

Figure 3.1 compares the errors for the different maximal Padé degrees. It shows a clear trend that the smaller the $m_{\max }$, the larger the error. The solid line is the unit roundoff multiplied by the (relative) condition number

$$
\operatorname{cond}(A)=\lim _{\epsilon \rightarrow 0} \max _{\|E\|_{2} \leq \epsilon\|A\|_{2}} \frac{\left\|e^{A+E}-e^{A}\right\|_{2}}{\epsilon\left\|e^{A}\right\|_{2}},
$$

which we estimate using the finite-difference power method of Kenney and Laub [23], [21, sect. 3.4]. For a method to perform in a backward stable, and hence forward stable, manner, its error should lie not far above this line on the graph. In all our figures the results are sorted by decreasing condition number cond $(A)$. We see that Algorithm $2.3\left(m_{\max }=13\right)$ performs in a numerically stable way on this experiment, even though two of the test matrices were chosen to cause the scaling and squaring method to "overscale" - a phenomenon investigated in [6] and [24]. Some instability is apparent for the smaller $m_{\max }$. The numerical results therefore concur with the theory in suggesting that the fewer the number of squarings, the smaller the error.

Figure 3.2 compares Algorithm 2.3 with expm, Sidje's function padm, and the MATLAB function funm, which implements the Schur-Parlett method of Davies and Higham [4], [21, Chap. 9], which is designed for general $f$. The figure shows that expm exhibits minor instability on many of the test matrices.

Finally, Figure 3.3 plots a performance profile [7], [18, sect. 22.4] for the experiment. Each of the methods is represented by a curve on the plot. For a given $\alpha$ on the $x$-axis, the $y$-coordinate of the corresponding point on the curve is the probability that the method in question has an error within a factor $\alpha$ of the smallest error over all 


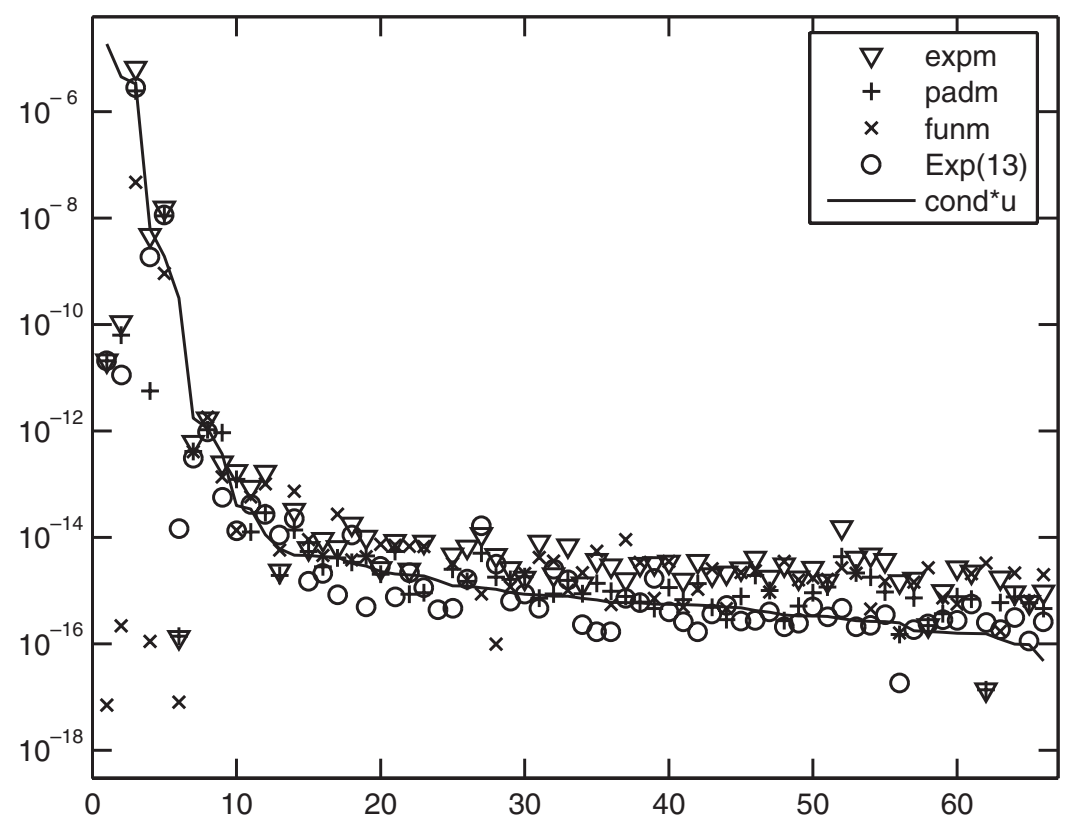

Fig. 3.2 Normwise relative errors for expm, padm (Sidje), funm, and Algorithm 2.3 (Exp(13)).

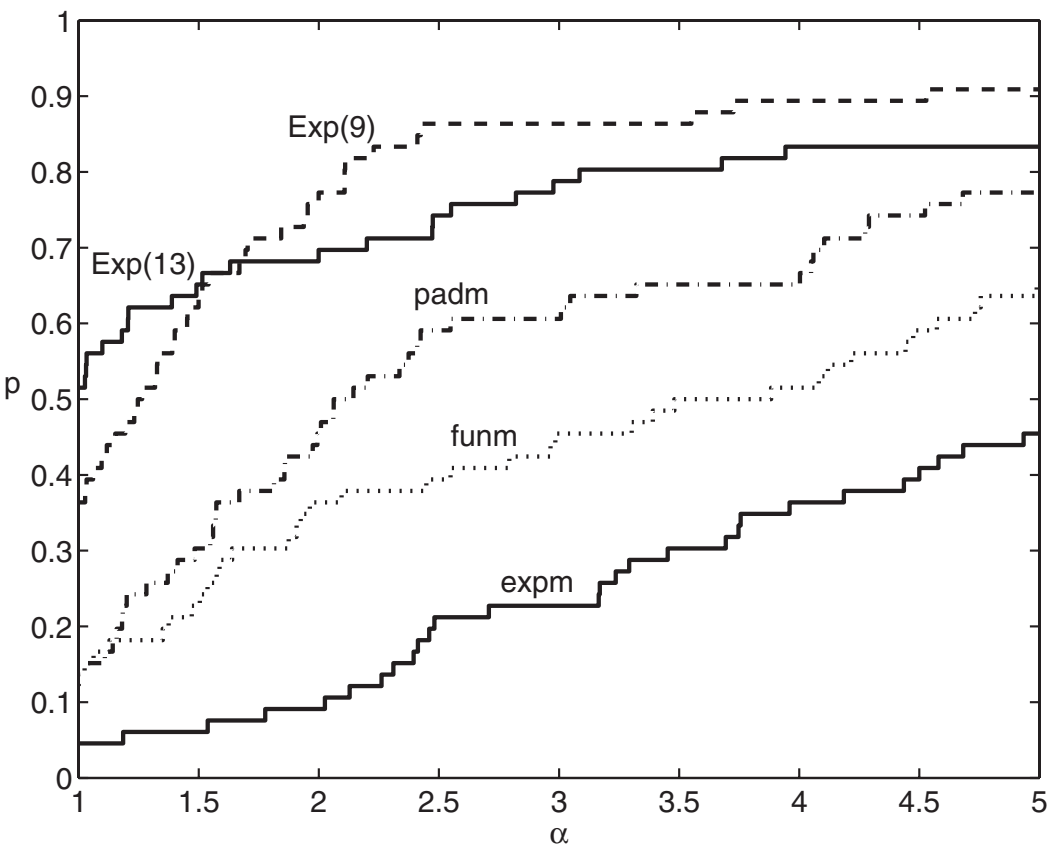

Fig. 3.3 Performance profile: $\alpha$ is plotted against the probability $p$ that a method has error within a factor $\alpha$ of the smallest error over all methods.

Copyright (c) by SIAM. Unauthorized reproduction of this article is prohibited. 
the methods, where probabilities are defined over the set of test problems. For $\alpha=1$, the $\operatorname{Exp}(13)$ curve is the highest: it intersects the $y$-axis at $p=0.52$, which means that this method has the smallest error in $52 \%$ of the examples - more often than any other method. For $\alpha \gtrsim 1.6, \operatorname{Exp}(9)$ is more likely than $\operatorname{Exp}(13)$ to be within a factor $\alpha$ of the smallest error. Since the curve for expm lies below all the other curves, expm is the least accurate method on this set of test matrices, as measured by the performance profile. Recall that the functions expm and padm both use $m=6$ and differ only in how they evaluate $r_{6}$, as described at the start of this section.

In interpreting the results it is worth noting that the actual errors the methods produce are sensitive to the details of the arithmetic. The version of Figure 3.3 produced by a prerelease version of MATLAB 7.0 was different, though qualitatively similar. (For example, the $\operatorname{Exp}(13)$ and $\operatorname{Exp}(9)$ curves touched at $\alpha=3$, though they did not cross.)

This experiment shows that in terms of accuracy in floating point arithmetic there is no clear reason to favor $\operatorname{Exp}(13)$ over $\operatorname{Exp}(9)$ or vice versa. Our choice of $\operatorname{Exp}(13)$ in Algorithm 2.3 on the grounds of its lower cost is therefore justified.

4. Indirect Padé Approximation. Najfeld and Havel [30, sect. 2] suggest an interesting variation of the standard scaling and squaring method that they claim is more efficient. Instead of approximating the exponential directly, they use a Padé approximation to the even function

$$
\begin{aligned}
\tau(x) & =x \operatorname{coth}(x)=x\left(e^{2 x}+1\right)\left(e^{2 x}-1\right)^{-1} \\
& =1+\frac{x^{2}}{3+\frac{x^{2}}{5+\frac{x^{2}}{7+\cdots}}}
\end{aligned}
$$

in terms of which the exponential can be written

$$
e^{2 x}=\frac{\tau(x)+x}{\tau(x)-x}
$$

The Padé approximants to $\tau$ can be obtained by truncating the continued fraction expansion (4.1). For example, using $\widetilde{r}_{2 m}$ to denote the diagonal $[2 m / 2 m]$ Padé approximant to $\tau$,

$$
\widetilde{r}_{8}(x)=\frac{\frac{1}{765765} x^{8}+\frac{4}{9945} x^{6}+\frac{7}{255} x^{4}+\frac{8}{17} x^{2}+1}{\frac{1}{34459425} x^{8}+\frac{2}{69615} x^{6}+\frac{1}{255} x^{4}+\frac{7}{51} x^{2}+1} .
$$

The numerators and denominators of $\widetilde{r}_{2 m}$ comprise only even powers of $x$, and so they can be evaluated at a matrix argument $A$ in $m$ matrix multiplications by explicitly forming the required even powers.

The error in $r_{2 m}$ has the form

$$
\tau(x)-\widetilde{r}_{2 m}(x)=\sum_{k=1}^{\infty} d_{k} x^{4 m+2 k}=\sum_{k=1}^{\infty} d_{k}\left(x^{2}\right)^{2 m+k} .
$$

(The error is one order in $x$ higher than the definition of Padé approximant requires, due to the fact that $\tau$ is even.) Hence the error in the matrix approximation satisfies

$$
\left\|\tau(A)-\widetilde{r}_{2 m}(A)\right\| \leq \sum_{k=1}^{\infty} d_{k}\left\|A^{2}\right\|^{2 m+k}=: \omega_{2 m}\left(\left\|A^{2}\right\|\right) .
$$


Let $\theta_{2 m}$ be the largest $\theta$ such that $\omega_{2 m}(\theta) \leq u$. The algorithm of Najfeld and Havel scales $\widetilde{A} \leftarrow A / 2^{s+1}$ with $s \geq 0$ chosen so that $\left\|\widetilde{A}^{2}\right\|=\left\|A^{2}\right\| / 2^{2 s+2} \leq \theta_{2 m}$. Padé approximation is applied to the scaled matrix, $\widetilde{A}$. The final stage consists of $s$ squarings, just as in the standard scaling and squaring method. Note that there are $s$ squarings rather than $s+1$, because the underlying approximation (4.2) is to $e^{2 x}$ and not $e^{x}$. Computation of the $\theta_{2 m}$ and analysis of computational cost in [30] leads Najfeld and Havel to conclude that the choice $m=8$ of Padé approximant degree leads to the most efficient algorithm.

Detailed study of this algorithm shows that it is competitive in cost with Algorithm 2.3. The following result reveals a close connection with Algorithm 2.3.

Theorem 4.1. The $[2 m / 2 m]$ Padé approximant $\widetilde{r}_{2 m}(x)$ to $x \operatorname{coth}(x)$ is related to the $[2 m+1 / 2 m+1]$ Padé approximant $r_{2 m+1}(x)$ to $e^{x}$ by

$$
r_{2 m+1}(x)=\frac{\widetilde{r}_{2 m}(x / 2)+x / 2}{\widetilde{r}_{2 m}(x / 2)-x / 2} .
$$

Proof. By (4.3),

$$
e_{2 m}(x):=\tau(x)-\widetilde{r}_{2 m}(x)=O\left(x^{4 m+2}\right) .
$$

Then

$$
\begin{aligned}
g(x) & :=\frac{\widetilde{r}_{2 m}(x)+x}{\widetilde{r}_{2 m}(x)-x}=\frac{\tau(x)+x-e_{2 m}(x)}{\tau(x)-x-e_{2 m}(x)} \\
& =\frac{\tau(x)+x}{\tau(x)-x}\left[\frac{1-e_{2 m}(x) /(\tau(x)+x)}{1-e_{2 m}(x) /(\tau(x)-x)}\right] \\
& =e^{2 x}\left[1-\frac{e_{2 m}(x)}{\tau(x)+x}+\frac{e_{2 m}(x)}{\tau(x)-x}+O\left(e_{2 m}(x)^{2}\right)\right] \\
& =e^{2 x}\left[1+\frac{2 x e_{2 m}(x)}{(\tau(x)+x)(\tau(x)-x)}+O\left(e_{2 m}(x)^{2}\right)\right] \\
& =e^{2 x}\left(1+x O\left(e_{2 m}(x)\right)=e^{2 x}+O\left(x^{4 m+3}\right) .\right.
\end{aligned}
$$

Now $g(x)$ is a rational function with numerator and denominator both of degree at most $2 m+1$, and $g(x / 2)=e^{x}+O\left(x^{4 m+3}\right)$. By the uniqueness of Padé approximants to the exponential, $g(x / 2) \equiv r_{2 m+1}(x)$.

Hence the algorithm of Najfeld and Havel, which takes $m=8$, is implicitly using the same Padé approximant to $e^{x}$ as Algorithm 2.3 when the latter takes $m=9$. The difference is essentially in how $A$ is scaled prior to forming the approximant and in the precise formulae from which the approximant is computed. While the derivation of Najfeld and Havel's algorithm ensures that the error $\left\|\tau(A)-\widetilde{r}_{2 m}(A)\right\|$ is sufficiently small for the scaled $A$, what this implies about the error $e^{2 A}-\left(\widetilde{r}_{2 m}(A)+\right.$ $A)\left(\widetilde{r}_{2 m}(A)-A\right)^{-1}$ is unclear, particularly since the matrix $\widetilde{r}_{2 m}(A)-A$ that is inverted can be arbitrarily ill conditioned. Moreover, it is unclear how to derive an analogue of Theorem 2.1 that expresses the truncation errors in the Padé approximant to $\tau$ as backward errors in the original data.

We conclude that the algorithm suggested by Najfeld and Havel is essentially a variation of the standard scaling and squaring method with direct Padé approximation but with weaker guarantees concerning its behavior both in exact arithmetic (since a backward error result is lacking) and in floating point arithmetic (since a possibly ill-conditioned matrix must be inverted). Without stronger supporting analysis the method cannot therefore be recommended. 
5. Conclusions. The scaling and squaring method has long been the most popular method for computing the matrix exponential. By analyzing it afresh we have found that existing implementations of Sidje [31] and Ward [38], and in the function expm in MATLAB 7.0, are not optimal. While they do guarantee a backward error of order the unit roundoff in the absence of roundoff (that is, solely considering truncation errors in the Padé approximation), they use more matrix multiplications than necessary. By developing an essentially optimal backward error bound for the scaling and squaring method in exact arithmetic that depends on $A$ only through $\|A\|$, we have identified the most efficient choice of degree $m$ of Padé approximation and initial scaling for IEEE double precision arithmetic: $m=13$, as opposed to $m=6$ for expm and Sidje's algorithm and $m=8$ for Ward's algorithm, with scaling to ensure $\|A\| \leq 5.4$. A welcome side effect has been to reduce the amount of scaling, and hence the number of squarings in the final stage. This reduction, together with a careful evaluation of the Padé approximation, makes the new algorithm typically more accurate than the old ones (see Figures 3.2 and 3.3).

With the aid of some new error analysis we have shown that all but one part of Algorithm 2.3 is numerically stable. The effect of rounding errors on the final squaring phase remains an open question, but in our experiments the overall algorithm has performed in a numerically stable way throughout.

Acknowledgments. I am grateful to Philip Davies for insightful comments on section 4 and Roy Mathias for suggesting evaluation schemes of the form (2.9).

\section{REFERENCES}

[1] A. H. Al-Mohy and N. J. Higham, Computing the Fréchet derivative of the matrix exponential, with an application to condition number estimation, SIAM J. Matrix Anal. Appl., 30 (2009), pp. 1639-1657.

[2] A. H. Al-Mohy AND N. J. Higham, A new scaling and squaring algorithm for the matrix exponential, SIAM J. Matrix Anal. Appl., 30 (2009), pp. 970-989.

[3] C. Brezinski and J. Van Iseghem, A taste of Padé approximation, Acta Numerica, 4 (1995), pp. 53-103.

[4] P. I. Davies and N. J. Higham, A Schur-Parlett algorithm for computing matrix functions, SIAM J. Matrix Anal. Appl., 25 (2003), pp. 464-485.

[5] I. S. Dhillon AND B. N. PARLETt, Orthogonal eigenvectors and relative gaps, SIAM J. Matrix Anal. Appl., 25 (2004), pp. 858-899.

[6] L. Dieci AND A. PAPINI, Padé approximation for the exponential of a block triangular matrix, Linear Algebra Appl., 308 (2000), pp. 183-202.

[7] E. D. Dolan And J. J. MoRÉ, Benchmarking optimization software with performance profiles, Math. Program., 91 (2002), pp. 201-213.

[8] E. Estrada and N. Hatano, Communicability in complex networks, Phys. Rev. E, 77 (2008), article 036111.

[9] E. Estrada And D. J. Higham, Network Properties Revealed through Matrix Functions, Mathematics Research Report 17, University of Strathclyde, Scotland, UK, 2008.

[10] E. Estrada, D. J. Higham, and N. Hatano, Communicability betweenness in complex networks, Phys. A, 388 (2009), pp. 764-774.

[11] E. Estrada and J. A. RodríGuez-Velázquez, Subgraph centrality in complex networks, Phys. Rev. E, 71 (2005), article 056103.

[12] G. F. Franklin, J. D. Powell, and M. L. Workman, Digital Control of Dynamic Systems, 3rd ed., Addison-Wesley, Reading, MA, 1998.

[13] R. A. Frazer, W. J. Duncan, And A. R. Collar, Elementary Matrices and Some Applications to Dynamics and Differential Equations, Cambridge University Press, Cambridge, UK, 1963.

[14] W. Gautschi, Numerical Analysis: An Introduction, Birkhäuser Boston, Boston, MA, 1997.

[15] G. H. Golub and C. F. Van Loan, Matrix Computations, 3rd ed., The Johns Hopkins University Press, Baltimore, MD, 1996. 
[16] G. I. Hargreaves and N. J. Higham, Efficient algorithms for the matrix cosine and sine, Numer. Algorithms, 40 (2005), pp. 383-400.

[17] T. F. Havel, I. NaJfeld, AND J. YAng, Matrix decompositions of two-dimensional nuclear magnetic resonance spectra, Proc. Natl. Acad. Sci. USA, 91 (1994), pp. 7962-7966.

[18] D. J. Higham and N. J. Higham, MATLAB Guide, 2nd ed., SIAM, Philadelphia, PA, 2005.

[19] N. J. Higham, The Matrix Computation Toolbox, http://www.ma.man.ac.uk/ ${ }^{\text {higham/ }}$ metoolbox.

[20] N. J. Higham, Accuracy and Stability of Numerical Algorithms, 2nd ed., SIAM, Philadelphia, PA, 2002.

[21] N. J. Higham, Functions of Matrices: Theory and Computation, SIAM, Philadelphia, PA, 2008.

[22] R. A. Horn and C. R. Johnson, Topics in Matrix Analysis, Cambridge University Press, Cambridge, UK, 1991.

[23] C. S. Kenney and A. J. Laub, Condition estimates for matrix functions, SIAM J. Matrix Anal. Appl., 10 (1989), pp. 191-209.

[24] C. S. Kenney AND A. J. LAUB, A Schur-Fréchet algorithm for computing the logarithm and exponential of a matrix, SIAM J. Matrix Anal. Appl., 19 (1998), pp. 640-663.

[25] S. KOIKARI, An error analysis of the modified scaling and squaring method, Comput. Math. Appl., 53 (2007), pp. 1293-1305.

[26] E. N. Laguerre, Le calcul des systèmes linéaires, extrait d'une lettre adressé à M. Hermite, in Oeuvres de Laguerre, Vol. 1, C. Hermite, H. Poincaré, and E. Rouché, eds., GauthierVillars, Paris, 1898, pp. 221-267. The article is dated 1867 and is "Extrait du Journal de l'École Polytechnique, LXII ${ }^{\mathrm{e}}$ Cahier."

[27] J. D. LaWson, Generalized Runge-Kutta processes for stable systems with large Lipschitz constants, SIAM J. Numer. Anal., 4 (1967), pp. 372-380.

[28] C. B. Moler And C. F. VAN LOAN, Nineteen dubious ways to compute the exponential of a matrix, SIAM Rev., 20 (1978), pp. 801-836.

[29] C. B. Moler and C. F. Van Loan, Nineteen dubious ways to compute the exponential of a matrix, twenty-five years later, SIAM Rev., 45 (2003), pp. 3-49.

[30] I. Najfeld And T. F. Havel, Derivatives of the matrix exponential and their computation, Adv. in Appl. Math., 16 (1995), pp. 321-375.

[31] R. B. SIDJE, Expokit: A software package for computing matrix exponentials, ACM Trans. Math. Software, 24 (1998), pp. 130-156.

[32] R. B. Sidje AND W. J. Stewart, A numerical study of large sparse matrix exponentials arising in Markov chains, Comput. Statist. Data Anal., 29 (1999), pp. 345-368.

[33] B. Skaflestad And W. M. Wright, The scaling and modified squaring method for matrix functions related to the exponential, Appl. Numer. Math., 59 (2009), pp. 783-799.

[34] M. Sofroniou And G. Spaletta, Efficient matrix polynomial computation and application to the matrix exponential, talk given at the workshop on Dynamical Systems on Matrix Manifolds: Numerical Methods and Applications, Bari, Italy, 2004.

[35] C. F. Van Loan, A Study of the Matrix Exponential, Numerical Analysis Report 10, The University of Manchester, Manchester, UK, 1975. Reissued as MIMS EPrint 2006.397, Manchester Institute for Mathematical Sciences, The University of Manchester, Manchester, UK, 2006.

[36] C. F. VAN LOAN, On the limitation and application of Padé approximation to the matrix exponential, in Padé and Rational Approximation: Theory and Applications, E. B. Saff and R. S. Varga, eds., Academic Press, New York, 1977, pp. 439-448.

[37] R. S. VARga, Matrix Iterative Analysis, 2nd ed., Springer-Verlag, Berlin, 2000.

[38] R. C. WARD, Numerical computation of the matrix exponential with accuracy estimate, SIAM J. Numer. Anal., 14 (1977), pp. 600-610.

Copyright (c) by SIAM. Unauthorized reproduction of this article is prohibited. 